\title{
Bone mineral density in rheumatoid arthritis patients 1 year after adalimumab therapy: arrest of bone loss
}

\author{
C A Wijbrandts, ${ }^{1}$ R Klaasen, ${ }^{1}$ M G W Dijkgraaf, ${ }^{2}$ D M Gerlag, ${ }^{1}$ B L F van Eck-Smit, ${ }^{3}$ \\ P P Tak'
}

${ }^{1}$ Division of Clinical Immunology and Rheumatology, Academic Medical Centre, University of Amsterdam, Amsterdam, The Netherlands; ${ }^{2}$ Department of Clinical Epidemiology, Biostatistics and Bioinformatics, Academic Medical Centre, University of Amsterdam, Amsterdam, The Netherlands; ${ }^{3}$ Department of Nuclear Medicine, Academic Medical Centre, University of Amsterdam, Amsterdam, The Netherlands

Correspondence to: P P Tak, Division of Clinical Immunology and Rheumatology, Academic Medical Centre, University of Amsterdam, Meibergdreef 9, 1105 AZ Amsterdam, The Netherlands; P.P.Tak@amc.uva.n

Accepted 31 March 2008 Published Online First 13 April 2008

\section{ABSTRACT}

Objective: To explore the effects of anti-tumour necrosis factor (TNF) $\alpha$ antibody therapy on bone mineral density (BMD) of the lumbar spine and femur neck in patients with rheumatoid arthritis (RA).

Methods: A total of 50 patients with active RA (DAS28 $\geqslant 3.2$ ) who started adalimumab (40 mg subcutaneously/2 weeks) were included in an open label prospective study. All patients used stable methotrexate and were allowed to use prednisone ( $\leqslant 10 \mathrm{mg} /$ day). The BMD of the lumbar spine and femur neck was measured before and 1 year after start of treatment.

Results: Disease activity at baseline (28-joint Disease Activity Score (DAS28)) and disease duration were inversely correlated with femoral neck BMD and lumbar spine BMD $(p<0.05)$. Mean BMD of lumbar spine and femur neck remained unchanged after 1 year of adalimumab therapy $(+0.3 \%$ and $+0.3 \%$, respectively). Of interest, a beneficial effect of prednisone on change in femur neck BMD was observed with a relative increase with prednisone use $(+2.5 \%)$ compared to no concomitant prednisone use $(-0.7 \%),(p=0.015)$.

Conclusion: In contrast to the progressive bone loss observed after conventional disease-modifying antirheumatic drug therapy, TNF blockade may result in an arrest of general bone loss. Consistent with previous observations, the data also suggest that the net effect of lowdose corticosteroids on BMD in RA may be beneficial, possibly resulting from their anti-inflammatory effects.

Osteoporosis is more frequent in patients with rheumatoid arthritis (RA) than in the general population due to active systemic inflammation as well as the use of corticosteroids and immobility. ${ }^{1}$ Osteoporosis can cause pain, loss of height and may lead to fractures after falling. Subsequent disability may lead to loss of independence and quality of life. Fractures and associated comorbidity may eventually even be life threatening in elderly patients. Hence, understanding and preventing osteoporosis in patients with RA is important, especially because the age of onset of RA is usually around the age of $50-60$ and twothirds of patients are female and past menopause, so already at risk of developing osteoporosis.

Tumour necrosis factor (TNF) is a key player in the pathogenesis of RA. TNF $\alpha$ is not only associated with inflammation, but also involved in postmenopausal bone loss. ${ }^{2}{ }^{3}$ Previous work in animal models of RA has suggested that TNF blockade may result in inhibition of systemic bone loss. ${ }^{4}$ Data describing the effect of in vivo TNF $\alpha$ inhibition on general bone loss in patients with RA are still limited and restricted to infliximab treatment. ${ }^{6}$ Therefore, our aim was to explore the effects of adalimumab treatment on bone mineral density (BMD) of the lumbar spine and femoral neck in patients with RA in an open label, prospective study.

\section{Patients and methods}

A total of 50 patients with active RA (28-joint Disease Activity Score (DAS28) ?3.2) who started adalimumab treatment (40 mg subcutaneously every other week) were included in an open label prospective study. All patients used stable methotrexate (MTX) and were allowed to use prednisone ( $\leqslant 10 \mathrm{mg} /$ day) if stable at least 1 month before inclusion. MTX was kept stable throughout the study, prednisone dosage was allowed to be tapered after 16 weeks of treatment and the continuation of antiosteoporotic drugs was allowed. Disease activity was assessed by DAS28 at week 4, 16, 28, 40 and 52. Clinical response was defined by the European League Against Rheumatism (EULAR) response criteria.'

The BMD of the lumbar spine (L1-L4) and left femoral neck was measured before treatment and after 1 year by dual energy $x$ ray absorptiometry (DXA) (ODR 4500A; Hologic Inc., Waltham, Massachusetts, USA). BMD was defined as bone mineral content, divided by the surface of the projected bone area, expressed in $\mathrm{g} / \mathrm{cm}^{2}$. T-scores (an SD compared with the peak $\mathrm{BMD}$ value of an adult aged from 20 to 30 years) and Z-scores (an $\mathrm{SD}$ compared with $\mathrm{BMD}$ values of age and sex) were calculated. Reference BMD values supplied by the manufacturer were used. Osteopoenia was defined by a $\mathrm{T}$-score between -1 and $-2.5 \mathrm{SD}$ and osteoporosis as a T-score below $-2.5 \mathrm{SD}$, according to the World Health Organization guidelines. The protocol was approved by the Medical Ethics Committee of the Academic Medical Centre, University of Amsterdam. All patients gave written informed consent.

\section{Statistical analysis}

Changes in BMD were analysed by paired samples $t$ tests. Subanalyses were performed by independent Student t tests or Mann-Whitney $U$ tests to detect significant differences in baseline DXA values and changes in $\mathrm{BMD}$ between groups including differences in prednisone or antiosteoporotic drug use. Correlations were assessed with the Pearson product-moment or Spearman rankorder correlation coefficients, whichever was appropriate. Stepwise backward multivariate linear regression analysis was performed on the total online under the BMJ Journals unlocked scheme, see http:// ard.bmj.com/info/unlocked.dt 
group to study the influence of antiosteoporotic drugs (calcium, vitamin $\mathrm{D}$ and biphosphonate), prednisone use and baseline $\mathrm{BMD}$ on change in femur BMD. Values are presented as mean (SD) or median and interquartile range (IOR). p Values $<0.05$ were considered statistically significant. SPSS V. 12.0.2 for Windows (SPSS, Chicago, Illinois, USA) was used.

\section{RESULTS}

\section{Patients and clinical response}

After 1 year of follow-up, 46 out of 50 patients completed the whole study, while 4 patients were excluded from analysis due to premature drop out (1 lost to follow-up, 1 infection, 1 lack of efficacy and 1 malignancy). In one patient the BMD of the femur neck was not performed after treatment due to hip replacement. A total of $80 \%$ were female and the mean age 51 (14) years. The median disease duration was 60 months (IOR 36 to 142 ). Furthermore, 31 (67\%) patients had erosive disease, $65 \%$ was IgM-rheumatoid factor positive and $70 \%$ anti-cyclic citrullinated peptide (CCP) positive. The mean MTX dose was 19.1 (6.9) $\mathrm{mg} /$ week. A total of 13 (28\%) patients used concomitant prednisone at a mean dose of 7.4 (2.3) $\mathrm{mg} /$ day. Eight patients used prednisone $\geqslant 5.0-7.5 \mathrm{mg} /$ day and five patients used $>7.5-\leqslant 10.0 \mathrm{mg} /$ day). Of these 13 patients, 6 received bisphosphonate therapy and 2 patients used bisphosphonate without prednisone. There were four patients with a disease duration less than 1 year of whom only one used prednisone; all other prednisone users had longstanding RA. There were 13 patients that used calcium and vitamin D3 suppletion, of whom 9 were also treated with prednisone. In five patients prednisone was stopped after week 16 while eight patients still used low-dose prednisone $(<10 \mathrm{mg} /$ day) after 1 year. In all, 19 (51\%) women were menopausal, of whom only 1 used hormonal replacement therapy, and 7 women were not menopausal and used an oral contraceptive (see table 1 for patient characteristics)

The mean DAS28 decreased from 5.4 (1.1) at baseline to 3.6 (1.2) at week 16 and $3.4(0.7)$ at week 52 (both p<0.001). At week 52 there were 23 good, 16 moderate and 7 non-responders according to the EULAR response criteria.

\section{BMD at baseline is associated with disease activity and disease duration}

Baseline mean lumbar spine BMD was $0.962(0.150) \mathrm{g} / \mathrm{cm}^{2}$ and mean femoral neck BMD was $0.798(0.156) \mathrm{g} / \mathrm{cm}^{2}$. Only 4 $(8.9 \%)$ of 46 analysed patients had osteoporosis. Osteopoenia was found in 13 (28\%) patients in femur neck and spine and in 4 patients in only the spine.

Males had a significantly higher BMD of the femoral neck than females $(p=0.011)$ with a similar trend for the lumbar spine $(p=0.062)$. There was a significant inverse correlation between $\mathrm{BMD}$ and age and, as expected, disease activity at baseline (DAS28) and disease duration were inversely correlated with femoral neck BMD ( $r=-0.36, p=0.015$ and $r=-0.37$, $p=0.011$, respectively). Lumbar spine BMD was also inversely correlated with the DAS28 and disease duration $(r=-0.44$ $p=0.003$ and $r=-0.33 p=0.029$, respectively). Of interest, only $\mathrm{BMD}$ of the femur was correlated with body weight $(r=0.438, p=0.002)$. There was no difference in baseline BMD between users and non-users of prednisone. A difference however was found in baseline $\mathrm{BMD}$ of lumbar spine and femur related to calcium, vitamin D3 or bisphosphonate use, with lower baseline BMD values for patients using antiosteoporotic therapy. Naturally, their low BMD was the reason why
Table 1 Baseline patient characteristics

\begin{tabular}{lc}
\hline Characteristic & Total $\mathbf{n}=\mathbf{4 6}$ \\
\hline Demographics: & \\
Age (years) & $51(14)$ \\
Female (\%) & $37(80)$ \\
BMI (kg/m²) & $27.0(5.9)$ \\
Disease status: & $60(36$ to 142$)$ \\
Disease duration (months) & $31(67)$ \\
Erosive disease (\%) & $30(65)$ \\
IgM-RF+(\%) & $32(70)$ \\
Anti-CCP+(\%) & $5.4(1.1)$ \\
DAS28 & $19(11$ to 34$)$ \\
ESR (mm/h) & $7(4$ to 17$)$ \\
CRP (mg/litre) & $19(51)$ \\
Postmenopausal women (\%) & \\
Drug treatments: & $4.1(2.0)$ \\
Previous DMARDs & $19.1(6.9)$ \\
Methotrexate (mg/week) & $13(28)$ \\
Prednisone use (\%) & $7.4(2.3)$ \\
Prednisone dose (mg/day) & $8(17)$ \\
Bisphosphonate use (\%) & $13(28)$ \\
Calcium use (\%) & $12(26)$ \\
Vitamin D use (\%) & \\
Bone mineral density: & \\
Lumbar spine & \\
BMD (g/cm²) & $0.96(0.15)$ \\
T-score (SD) & $-0.82(1.32)$ \\
Z-score (SD) & $0.04(1.23)$ \\
Femoral neck & \\
BMD (g/cm²) & $0.80(0.16)$ \\
T-score (SD) & $0.19(1.19)$ \\
Z-score (SD) & \\
\hline Men & \\
&
\end{tabular}

Mean values (SD), median and interquartile range (IQR) or percentages are shown. $\mathrm{BMD}$, bone mineral density; BMI, body mass index; CCP, cyclic citrullinated peptide; CRP, C-reactive protein; DAS28, 28-joint Disease Activity Score; DMARD, diseasemodifying antirheumatic drug; ESR, erythrocyte sedimentation rate; RF, rheumatoid factor.

these patients were using antiosteoporotic drugs to begin with. When comparing the presence or absence of erosions, rheumatoid factor or anti-CCP positivity we found no relationship with pretreatment $\mathrm{BMD}$.

\section{Further decrease in BMD is arrested by adalimumab therapy}

There was no significant change in mean BMD of the lumbar spine comparing values before $\left(0.962(0.150) \mathrm{g} / \mathrm{cm}^{2}\right)$ vs values after 1 year $\left(0.965(0.155) \mathrm{g} / \mathrm{cm}^{2}\right)(+0.3 \%)$ (table 2$)$. The mean $\mathrm{BMD}$ of the femur neck also remained unchanged: 0.798 $(0.156) \mathrm{g} / \mathrm{cm}^{2}$ before compared to $0.800(0.147) \mathrm{g} / \mathrm{cm}^{2}$ after 1 year of adalimumab therapy $(+0.3 \%)$ (see fig 1$)$. Hence, there was no significant increase or decrease after 1 year of treatment.

\section{The increase in BMD is related to the decrease in serum CRP levels}

An association was found between the decrease in serum CRP levels at week 16 and the increase in femur neck BMD after 1 year (Pearson correlation coefficient $r=-0.307, p=0.040$ ). In addition, a possible association, just falling short of statistical significance, was observed between the decrease in serum CRP levels at week 52 and the increase in femur neck BMD at the same time point (Pearson correlation coefficient $r=-0.292$, $\mathrm{p}=0.052)$. 


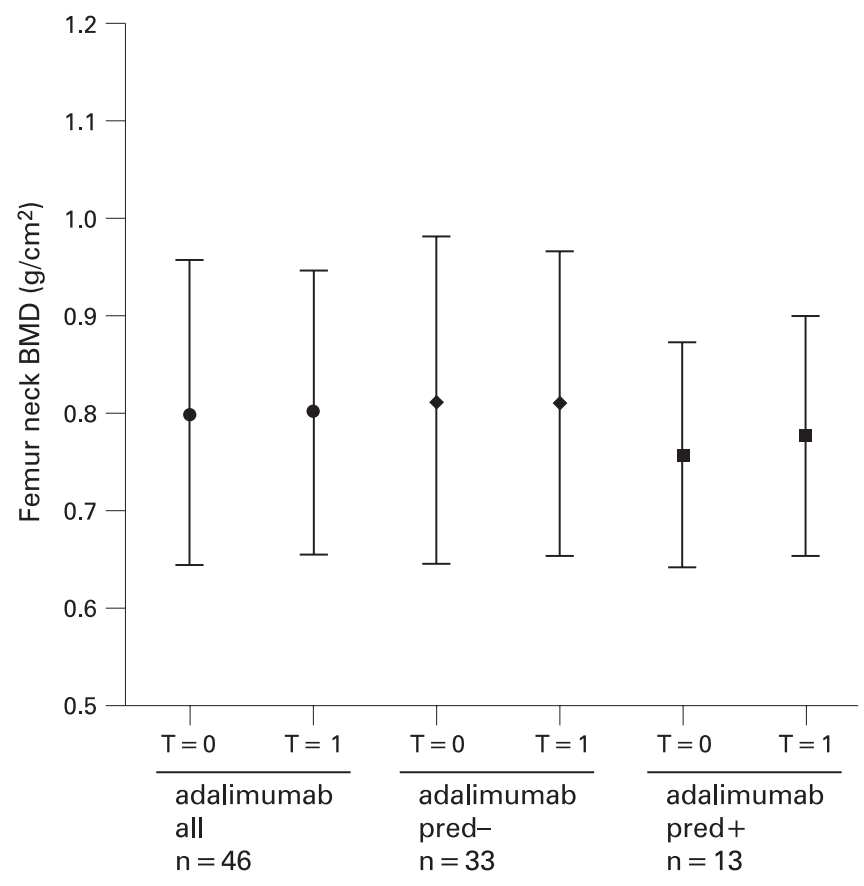

Figure 1 Change in femur neck bone mineral density (BMD) after 1 year of adalimumab therapy. Data are represented as mean (symbol) and SD (whiskers). After 1 year $(T=1)$ of adalimumab therapy the mean femur neck BMD was unchanged $(+0.3 \%)(n=46$, circle). However, the concomitant use of prednisone led to a relative increase in mean femur neck BMD $(+2.5 \%)(n=13$, square) compared to patients who did not use prednisone $(-0.7 \%)(n=33$, diamond).

\section{A favourable change in femur BMD in concomitant prednisone users}

Interestingly, univariate analysis indicated that the concomitant use of prednisone, calcium and vitamin D3 was associated with a greater increase in femur BMD compared to patients who did not use these drugs in combination with adalimumab. Especially, mean femur BMD showed a relative increase when prednisone was used: before $0.758(0.117)$ vs after 1 year 0.777 (0.122) ( $+2.5 \%)$ compared to no concomitant prednisone use $0.814(0.167)$ vs $0.808(0.126)(-0.7 \%),(p=0.015$, see fig 1$)$. A trend was observed for changes in lumbar spine $\mathrm{BMD}$ $(p=0.072)$. Although this was statistically significant for all three drugs, this exploratory study was not formally powered to study significant differences in mean change between groups. In response to this interesting observation, we performed stepwise backward multivariate linear regression analysis to study how much of the change in femur BMD independent of the overall effect of adalimumab could be explained by the use of these drugs. Interaction between prednisone, bisphosphonate, calcium and vitamin D use was studied by using an interaction variable in the analysis. We found that the pretreatment femur neck BMD $(p=0.035)$ and the use of prednisone $(p=0.031)$ together explained $18.5 \%\left(\right.$ adjusted $\mathrm{R}^{2}$ ) of the variance in $\mathrm{BMD}$ change.

Only the use of vitamin D3 showed an interaction with prednisone use. However, by including baseline femur BMD in the model vitamin $\mathrm{D}$ was rejected, because vitamin $\mathrm{D}$ was associated with a lower mean femur BMD pretreatment (vitamin D3 use 0.659 (0.104) vs no vitamin D3 use 0.847 (0.141), $\mathrm{p}<0.001)$, while prednisone use was unrelated to baseline femur BMD. Hence, these data suggest that in addition to the overall effect of adalimumab in all patients, $18.5 \%$ of the change in $\mathrm{BMD}$ of the femur is explained by baseline $\mathrm{BMD}$ and prednisone use.

\section{DISCUSSION}

The results of this study confirm and extend previous findings with infliximab in RA. We observed that BMD does not increase significantly after 1 year of adalimumab therapy, but on average progressive bone loss is halted.

Several studies have been performed with infliximab to study the effect of TNF $\alpha$ blockade on general bone loss in RA and ankylosing spondylitis. ${ }^{5-10}$ The results vary by study with regard to the magnitude of observed change, but also with regard to the time points of DXA scanning, the numbers of patients included and the concomitant use of prednisone and/or antiosteoporotic drugs. Our results are consistent with the findings of two previous studies on the effects of infliximab where bone mineral density was measured after 1 year and concomitant use of steroids and bisphosphonate was also allowed. The results from both studies suggest an arrest in further bone loss after TNF blockade. ${ }^{59}$ However, one of the latter studies showed that bone loss of the hands as measured by radiogrammetry was ongoing despite TNF blockade. ${ }^{5}$

The limitation of our study and previous studies is the fact that the design is not double blind and placebo controlled, making it difficult to interpret the size of the effect of TNF blockade. Because observed changes in BMD are very small in most bone mineral density studies and the SD of $\mathrm{BMD}$ measurements is quite large, a simple power calculation would tell us that one would need a study including around at least 500 patients to detect a significant change in BMD that is greater than the variance in $\mathrm{BMD}$ within the study group. However, a recent study used an elegant alternative by describing the change in $\mathrm{BMD}$ of femur and lumbar spine in a control group of 99 patients with RA using no TNF blocker but only methotrexate. ${ }^{9}$ The disease duration was comparable to the study group comprising 90 patients using infliximab in combination with methotrexate. Similar to our study, concomitant use of prednisone and bisphosphonates was allowed, resembling the normal RA population seen in the everyday rheumatology practice. Their results showed that within 1 year of follow-up the BMD of lumbar spine as well as the femur $\mathrm{BMD}$ decreased in the control group $(-3.9 \%$ and $-2.5 \%$, respectively) while mean lumbar spine and femur $\mathrm{BMD}$ remained unchanged in 90 patients using infliximab in

Table 2 Femur neck and lumbar spine bone mineral density (BMD) before and after 1 year

\begin{tabular}{lllllll}
\hline & BMD T $=\mathbf{0}$ & & & BMD T $=1$ year & \\
\cline { 2 - 3 } & Median (IOR) & Mean (SD) & & Median (IOR) & Mean (SD) & p Value \\
\hline $\begin{array}{l}\text { Femur } \\
\text { neck }\end{array}$ & $0.795(0.703$ to 0.920$)$ & $0.798(0.156)$ & & $0.798(0.685$ to 0.901$)$ & $0.800(0.147)$ & NS \\
$\begin{array}{l}\text { Lumbar } \\
\text { spine }\end{array}$ & $0.961(0.850$ to 1.070$)$ & $0.962(0.150)$ & & $0.963(0.845$ to 1.070$)$ & $0.965(0.155)$ & NS \\
\hline
\end{tabular}

BMD, bone mineral density; IQR, interquartile range; NS, not significant. 
combination with methotrexate. Historical data from a population study with patients with RA on traditional DMARD therapy (48\% using corticosteroids and 37\% using antiresorptive drugs) showed mean bone loss of the spine to be around $-0.29 \%$ and of the femur neck around $-0.64 \%$ after 1 year. In patients not using antiresorptive therapy but calcium and vitamin $\mathrm{D}$ alone bone loss of the spine was around $-1.99 \%$ and $-1.39 \%$ of the femur neck after 1 year. ${ }^{1}$ In early arthritis patients the percentage of bone loss is even higher $(-2.4 \%$ in the spine and $-4.3 \%$ in the trochanter after 1 year $).{ }^{11}$ With this perspective the results observed after anti-TNF $\alpha$ therapy suggest a favourable effect on generalised BMD.

This brings up the unexpected observation in our study of a potentially beneficial effect of prednisone on $\mathrm{BMD}$ change of the femur neck, for prednisone use is obviously more often associated with loss of bone mineral density. Corticosteroid use has been shown to be an independent risk factor for vertebral fractures. ${ }^{12}$ Recently however a placebo controlled trial found a positive effect of prednisone on hand bone density compared to placebo in early RA. ${ }^{13}$ Hence, prednisone can have a negative effect on bone mineral density when chronically used in high dose, but the beneficial effect of prednisone on further dampening inflammation and associated bone loss cannot be discarded. In addition, clinical improvement due to prednisone use and increased mobility due to clinical improvement may also have contributed to a positive effect on bone mineral density. The actual size of the potentially beneficial effect should further be determined in a placebo controlled study to rule out the impact of, for instance, regression to the mean, which might have exerted some influence in our cohort study, because baseline femur neck BMD was slightly lower in prednisone users. Of interest, in a recent study the effect of infliximab on femur BMD was no longer significant when steroid use was added to the multivariate model in addition to male sex and age. Although only an interaction between vitamin $\mathrm{D}$ suppletion and prednisone was identified by multivariate linear regression analysis, it cannot be completely ruled out that antiosteoporotic treatment in a subset of these patients added to the relative increase in bone mineral density of the femur neck. In summary this is the first study to show progressive general bone loss may also be arrested by adalimumab therapy. The data also suggest that the net effect of low-dose corticosteroids on BMD in RA may be beneficial, which may be explained by the anti-inflammatory effects.
Funding: DMG was supported by the Dutch Arthritis Association. This study was supported by Abbott Laboratories.

Competing interests: PPT is a member of the advisory board of Abbott and has received honoraria for lectures. The study sponsors had no involvement in the study design, the collection, analysis and interpretation of the data, writing the report, or the decision to submit the paper for publication.

Ethics approval: The protocol was approved by the Medical Ethics Committee of the Academic Medical Centre, University of Amsterdam. All patients gave written informed consent.

\section{REFERENCES}

1. Haugeberg G, Orstavik RE, Uhlig T, Falch JA, Halse JI, Kvien TK. Bone loss in patients with rheumatoid arthritis: results from a population-based cohort of 366 patients followed up for two years. Arthritis Rheum 2002;46:1720-8.

2. Ammann P, Rizzoli R, Bonjour JP, Bourrin S, Meyer JM, Vassalli P, et al. Transgenic mice expressing soluble tumor necrosis factor-receptor are protected against bone loss caused by estrogen deficiency. J Clin Invest 1997;99:1699-703.

3. Roggia C, Gao Y, Cenci S, Weitzmann MN, Toraldo G, Isaia G, et al. Up-regulation of TNF-producing $T$ cells in the bone marrow: a key mechanism by which estrogen deficiency induces bone loss in vivo. Proc Natl Acad Sci USA 2001;98:13960-5.

4. Saidenberg-Kermanac'h N, Corrado A, Lemeiter D, deVernejoul MC, Boissier MC, Cohen-Solal ME. TNF- $\alpha$ antibodies and osteoprotegerin decrease systemic bone loss associated with inflammation through distinct mechanisms in collagen-induced arthritis. Bone 2004;35:1200-7.

5. Vis M, Havaardsholm EA, Haugeberg G, Uhlig T, Voskuyl AE, van de Stadt RJ, et al. Evaluation of bone mineral density, bone metabolism, osteoprotegerin and receptor activator of the $\mathrm{NF} \kappa \mathrm{B}$ ligand serum levels during treatment with infliximab in patients with rheumatoid arthritis. Ann Rheum Dis 2006;65:1495-9.

6. Vis $\mathbf{M}$, Wolbink GJ, Lodder MC, Kostense PJ, van de Stadt RJ, de Koning MH, et al. Early changes in bone metabolism in rheumatoid arthritis patients treated with infliximab. Arthritis Rheum 2003;48:2996-7.

7. van Gestel AM, Anderson JJ, van Riel PL, Boers M, Haagsma CJ, Rich B, et al. ACR and EULAR improvement criteria have comparable validity in rheumatoid arthritis trials. American College of Rheumatology European League of Associations for Rheumatology. J Rheumatol 1999;26:705-11.

8. Lange U, Teichmann J, Muller-Ladner U, Strunk J. Increase in bone mineral density of patients with rheumatoid arthritis treated with anti-TNF- $\alpha$ antibody: a prospective open-label pilot study. Rheumatology (Oxford) 2005;44:1546-8.

9. Marotte H, Pallot-Prades B, Grange L, Gaudin P, Alexandre C, Miossec P. A 1-year case-control study in patients with rheumatoid arthritis indicates prevention of loss of bone mineral density in both responders and nonresponders to infliximab. Arthritis Res Ther 2007:9:R61.

10. Allali F, Breban M, Porcher R, Maillefert JF, Dougados M, Roux C. Increase in bone mineral density of patients with spondyloarthropathy treated with anti-tumour necrosis factor $\alpha$. Ann Rheum Dis 2003;62:347-9.

11. Gough AK, Lilley J, Eyre S, Holder RL, Emery P. Generalised bone loss in patients with early rheumatoid arthritis. Lancet 1994;344:23-7.

12. Ørstavik RE, Haugeberg G, Mowinckel P, Høiseth A, Uhlig T, Falch JA. Vertebral deformities in rheumatoid arthritis: a comparison with populationbased controls. Arch Intern Med 2004;164:420-5.

13. Haugeberg G, Strand A, Kvien TK, Kirwan JR. Reduced loss of hand bone density with prednisolone in early rheumatoid arthritis: results from a randomized placebocontrolled trial. Arch Intern Med 2005;165:1293-7. 